Published in final edited form as:

Anesthesiology. 2006 October ; 105(4): 660-664.

\title{
Can anesthetic technique for primary breast cancer surgery affect recurrence or metastasis?
}

\author{
Aristomenis K Exadaktylos, MD*, Donal J Buggy, MD, MSc,FRCPI, FCARCSI, FRCA ${ }^{\dagger}$, Denis \\ C Moriarty, FCARCSI§, Edward Mascha, PhD \\ * Research Fellow in Anaesthesia, Department of Anaesthesia, Mater Misericordiae University \\ Hospital, Dublin, Ireland \\ † Consultant in Anaesthesia, Mater Misericordiae University Hospital \& National Breast Screening \\ Programme (Eccles Unit); Honorary Senior Lecturer in Anaesthesia, University College Dublin, \\ Ireland \\ $\S$ Professor of Anaesthesia, Mater Misericordiae University Hospital \& University College Dublin, \\ Ireland \\ ‡ Statistician, Department of Quantitative Health Sciences, Cleveland Clinic Foundation, 9500 \\ Euclid Avenue, Cleveland, OH, 44195, USA \\ \# Chair, Department of OUTCOMES RESEARCH, Cleveland Clinic Foundation Cleveland, $\mathrm{OH}$; \\ Director, OUTCOMES RESEARCH Institute, and Weakley Professor of Anesthesiology, University \\ of Louisville, Louisville, $K Y$
}

\section{Abstract}

Background: Regional anesthesia is known to prevent or attenuate the surgical stress response; thus, inhibiting surgical stress by paravertebral anesthesia might attenuate perioperative factors that enhance tumor growth and spread. We hypothesized that breast cancer patients undergoing surgery with paravertebral anesthesia and analgesia combined with general anesthesia have a lower incidence of cancer recurrence or metastases than patients undergoing surgery with general anesthesia and patient-controlled morphine analgesia.

Methods: In this retrospective study, we examined the medical records of 129 consecutive patients undergoing mastectomy and axillary clearance for breast cancer between September 2001 and December 2002.

Results: 50 patients had surgery with paravertebral anesthesia and analgesia combined with general anesthesia and 79 patients had general anesthesia combined with postoperative morphine analgesia. The follow-up time was $32 \pm 5$ months (mean \pm SD). There were no significant differences in patients

Correspondence to: Donal J Buggy, Department of Anaesthesia, Mater Misericordiae University Hospital \& National Breast Screening Programme Eccles Unit, Dublin 7, Ireland. E-mail: donal.buggy @nbsp.ie. Ph:+353-1-8032281; Fax: 353-1-8309563. On the world wide web: www.mater.ie; www.or.org.

Funding details: Supported by The Mater College (Dublin Ireland), the National Institutes of Health Grant GM 061655 (Bethesda, MD), the Gheens Foundation (Louisville, KY), the Joseph Drown Foundation (Los Angeles, CA), and the Commonwealth of Kentucky Research Challenge Trust Fund (Louisville, KY). The sponsors did not influence any aspect of the study.

This work has not been previously published or submitted for publication.

Summary Statement: This retrospective analysis suggests that paravertebral anesthesia and analgesia for breast cancer surgery reduces the risk of recurrence or metastasis during the initial years of follow-up.

Submitted from: University Department of Anaesthesia \& Intensive Care Medicine, Mater Misericordiae University Hospital \& National Breast Screening Programme Eccles Unit, Dublin Ireland; Outcomes Research Institute, Louisville, Kentucky USA.

Summary Statement: This retrospective analysis suggests that paravertebral anesthesia and analgesia for breast cancer surgery reduces the risk of recurrence or metastasis during the initial years of follow-up. 
or surgical details, tumor presentation, or prognostic factors. Recurrence and metastasis-free survival was $94 \%(95 \%$ CI 87,100) and 82\% $(74,91)$ at 24 months and $94(87,100)$ and $77(68,87)$ at 36 months in the paravertebral and general anesthesia patients, respectively, $P=0.012$.

Conclusions: This retrospective analysis suggests that paravertebral anesthesia and analgesia for breast cancer surgery reduces the risk of recurrence or metastasis during the initial years of followup. Prospective trials evaluating the effects of regional analgesia and morphine sparing on cancer recurrence seem warranted.

\section{Introduction}

Chemotherapy, endocrine therapy, and radiotherapy all have significant roles in the treatment of breast cancer, but surgical removal of the tumor offers the best prospect for a good prognosis. ${ }^{1}$ However, the process of surgery inevitably induces a profound neuroendocrine, metabolic, and cytokine response. ${ }^{2} \mathrm{~A}$ consequence of this stress response is transient perioperative inhibition of immune function. ${ }^{3}$

Regional anesthesia is known to prevent or attenuate the surgical stress response by blocking afferent neural transmission, which prevents noxious afferent input from reaching the central nervous system. ${ }^{4}$ Paravertebral anesthesia is a regional anesthesia technique that is suitable for breast surgery when applied in the upper thoracic region. ${ }^{5}$ We have previously demonstrated its efficacy in suppressing the stress response to breast surgery, although without a concomitant reduction in factors associated with tumor cell angiogenesis. ${ }^{6}$ As importantly, paravertebral analgesia reduces the need for opioids which themselves impair immune function. 7,8

Experimental studies have shown that a number of perioperative factors, including regional anesthesia and optimum postoperative analgesia independently reduce the metastatic burden in animals inoculated with a strain of breast adenocarcinoma. ${ }^{9-11}$ It is plausible that inhibition of the surgical stress response by paravertebral anesthesia might attenuate perioperative factors that enhance tumor growth and spread. However, the effect of anesthetic technique on breast cancer outcome has not been evaluated in the clinical setting. In a retrospective analysis of existing medical records, we compared local recurrence and metastases in patients who had breast cancer surgery with and without paravertebral analgesia.

\section{Materials and Methods}

After approval by the Ethics Committee of the Mater Misericordiae University Hospital, Dublin Ireland, we reviewed the medical records of 165 patients who underwent mastectomy with axillary clearance or simple complete mastectomy between September 2001 and December 2002. Only the records from patients with a palpable breast lesion were included because the risk of recurrence is high in these patients. Furthermore, patients with smaller, screen-detected lesions tend to have less extensive operations that do not require paravertebral blocks and would have an extremely low incidence of recurrence over a longer time frame than we could review. We thus excluded patients who presented only for wide local excision with sentinel-node axillary sampling, patients with cancers detected by the National Breast Screening Programme (because screen-detected cancers are less severe), and patients having plastic or reconstructive surgery.

Paravertebral anesthesia was introduced to our department in September 2001. Local departmental policy was to offer it in combination with general anesthesia to patients undergoing extensive breast surgery involving axillary node clearance. In these patients, a catheter was inserted before to induction of anesthesia into the ipsilateral paravertebral space at the level of $\mathrm{T} 2$ or T 3 using a standard technique (paravertebral group). ${ }^{5}$ Each of these patients 
was given a $0.2-\mathrm{mL} \mathrm{kg}^{-1}$ bolus of levobupivacaine $0.25 \%$ before induction of general anesthesia. An infusion of plain levobupivacaine $0.25 \%$ was continued for $48 \mathrm{hr}$ postoperatively. The same anesthetist (DJB) placed all the paravertebral catheters; the same surgeon performed all the operations; and the same oncologist cared for all of the patients.

General anesthesia was induced with fentanyl $0.5 \mu \mathrm{g} \cdot \mathrm{kg}^{-1}$ and propofol $1.5-3.0 \mathrm{mg} \cdot \mathrm{kg}^{-1}$. After placement of a laryngeal mask airway, anesthesia was maintained with spontaneous breathing of sevoflurane 2-3\% in nitrous oxide and oxygen. All patients received diclofenac, $100 \mathrm{mg}$ rectally, preoperatively after induction of general anesthesia. Morphine $0.05 \mathrm{mg}^{-\mathrm{kg}^{-1}}$ boluses were given to patients in either group intraoperatively, at the discretion of the anesthetist.

All patients studied were under the care of the same surgeon. Patients cared for by anesthetists other than DJB were not offered paravertebral anesthesia because the technique had been introduced into our department only recently. These patients, and those who declined paravertebral anesthesia or in whom it was contraindicated, received balanced general anesthesia with postoperative patient-controlled morphine analgesia (general anesthesia group). Patient-controlled analgesia was administered via a CADD-Legacy ambulatory infusion pump (model number 6300, Deltec Inc, St Paul MN) using morphine bolus $1 \mathrm{mg}$, lockout time $6 \mathrm{~min}$.

The main outcome measure was the incidence of metastatic spread or cancer recurrence through August 2005 (i.e. follow-up interval of 2.5-4.0 years). We obtained the demographic data, details of the tumor size, grade, type, and estrogen receptor status; the extent of axillary nodal disease, whether postoperative or preoperative adjuvant chemotherapy or radiotherapy was used; and the patients' current status as determined by documentation of their follow up visits to the outpatient clinics or their general practitioners. Additionally, we assigned a Nottingham Prognostic Index, a score for which the prognosis for breast cancer is based on the formula: 0.2 (tumor size) + histological grade (1=Grade 1, least aggressive tumor appearance on histology, Grade $2=$ intermediate appearance, Grade $3=$ most aggressive appearance), + axillary lymph node involvement ( $1=$ no axillary lymph nodes involved, $2=$ up to 3 axillary lymph nodes involved, $3=$ more than 3 axillary lymph nodes involved). ${ }^{12}$ A score below 3.4 indicates a good outcome, and a score between 3.4 and 5.4 suggests an intermediate prognosis. In addition, we recorded the tumor margins, if the tumor was estrogen positive, and if the patient received chemotherapy, radiotherapy, or endocrine therapy. The patient-scored pain rating on the 11point Likert scale at $4 \mathrm{hr}$ and $24 \mathrm{hr}$ after surgery was also retrieved from the record.

If the most recent follow-up documentation in the hospital records exceeded 3 months from the date of our assessment, we contacted the patient's general practitioner by telephone to ensure that no consultation had occurred in the interim.

We recorded the data in coded form on an Excel spreadsheet and imported it into SAS statistical software (Carey, NC) for analysis. Normally distributed continuous variables were compared with independent sample t-tests and non-normal variables with Mann-Whitney U tests; categorical variables were compared with Chi-Square tests or Fisher Exact tests, as appropriate. A Kaplan-Meier log-rank test was used for univariable analysis and Cox proportional hazards regression was used for multivariable analysis of the time-to-recurrence of cancer between groups. Factors considered for the multivariable model were age, family history of cancer, tumor size, histological grade, duration of surgery, and blood loss. All factors significant at $\mathrm{P}<$ 0.25 were retained in the model. Associations with $\mathrm{P}<0.05$ were deemed statistically significant. 


\section{Results}

We reviewed the medical records of 129 patients who underwent mastectomy and axillary clearance between September 2001 and December 2002 and who met our inclusion criteria. An additional 65 patients who had surgery during that period did not qualify. Fifty qualifying patients had surgery with paravertebral anesthesia and general analgesia and 79 had general anesthesia with postoperative morphine analgesia. The follow-up time was $32 \pm 5$ months (mean \pm SD). There were no significant differences in patient characteristics. The median pain score was less in the paravertebral patients than in those receiving postoperative morphine analgesia (Table 1).

Paravertebral analgesia was deemed unsuccessful in two patients, one in recovery and one after 16 hours. These patients were given morphine patient-controlled analgesia, but their data were analysed as part in the paravertebral group. No other paravertebral patients required morphine. Three patients in the general anesthesia group developed infections (4\%), compared with two $(4 \%)$ in the paravertebral group. No patient in either group received a blood transfusion.

Preoperatively, none of the patients was taking oral opioids, and only one patient in each group was taking oral steroid therapy.

Tumor presentation and prognostic factors were similar in the two groups. Both groups had a mean Nottingham Prognostic Index score in the intermediate range. Moreover, the incidence of chemotherapy, radiotherapy, and endocrine therapy after the initial surgical treatment did not differ significantly between the groups (Table 2). Recurrence or metastasis was documented in 3 of 50 (6\%) patients in the paravertebral group and in 19 of 79 (24\%) patients in the general anesthesia group during the follow-up period (Table 3). When adjusting for the varied length of follow-up for each patient with a Kaplan-Meier analysis, the paravertebral group had slower time to recurrence $(P=0.013, \log$-rank test, Fig. 1). Furthermore, in a multivariable analysis adjusting for histological grade $(P=0.25)$ and axillary node involvement $(P=0.01)$, the risk of recurrence was significantly less in the paravertebral group $(P=0.012$, estimated hazards ratio $[95 \% \mathrm{CI}]$ of $0.21[0.06,0.71])$.

Only one patient, assigned to the general anesthesia group, died during the follow-up period. Both lung and bony metastases were found in this patient.

\section{Discussion}

Development of postoperative cancer recurrence and metastases may be analogous to development of postoperative surgical wound infection. All surgical wounds become contaminated with bacteria perioperatively. Whether contamination becomes a clinical infection depends critically on the patient's immune status. Furthermore, there is considerable evidence that the "decisive period" for establishment of clinical infections is largely restricted to the intraoperative period and subsequent few hours. ${ }^{13,14}$ Perioperative factors such as maintaining normothermia 15,16 or providing supplemental oxygen 17,18 thus modulate infection risk - even though infections are not detected clinically until days later.

As with the bacterial contamination that inevitably accompanies operations, surgery to remove primary breast cancer inadvertently disseminates tumor cells into both surrounding tissues and the systemic circulation. ${ }^{18}$ And as with the progression from bacterial contamination to clinical infection, progression from local or circulating tumor cells to clinical recurrence and metastases likely depends on the host defense mechanisms. ${ }^{9-11,19}$ Perioperative factors influencing immune competence may thus modulate the risk of recurrence or metastasis. $3,9-11$

Perioperative immune competence is impaired by several factors. The most obvious being surgery per se which elicits a profound neuroendocrine and cytokine flux. This stress response impairs numerous immune functions including a marked attenuation of natural killer cell 
function. ${ }^{9-11,16,19}$ Natural killer cells are thought to play a central role in preventing tumor dissemination and establishment. ${ }^{3}$ The stress response to surgery, is attenuated by regional anesthesia, including paravertebral anesthesia. ${ }^{2,6}$ Experimental studies in rats, for example, show that surgical stress is attenuated better by regional than by general anesthesia and that, consequently, natural killer cell function is better preserved and metastatic load to the lungs is reduced. ${ }^{9}$

A second factor influencing perioperative immune competence is anesthesia per se. Anesthetic drugs impair numerous immune functions, including neutrophil and natural killer cells. ${ }^{7,18,}$

20,21 Patients with concomitant regional anesthesia might require less general anesthesia, and thus perhaps have less anesthetic-induce immune impairment. But the major effect of regional analgesia is to spare patients postoperative opioids. This is an important effect since opioids, including the one most commonly used - morphine - inhibit both cellular and humoral immune function. ${ }^{7,8}$ Consequently, optimum analgesia helps preserve natural killer cell function in animals and reduces metastatic spread of cancer. ${ }^{10}$ Furthermore, morphine is proangiogenic and promotes release of survival-enhancing factors that augment breast tumor growth. 22

We speculate that regional anesthesia and analgesia thus might help maintain perioperative immune function both by reducing general anesthesia requirements and by sparing postoperative opioids. To the extent that paravertebral anesthesia and analgesia help maintain normal perioperative immune function, the technique seems likely to reduce the risk of tumor recurrence or metastasis. Our analysis is consistent with this theory: paravertebral anesthesia and analgesia for breast cancer surgery reduced the risk of recurrence or metastasis four-fold during a 2.5 to 4 -year follow-up period.

This study has several important limitations inherent in its retrospective, observational design. Patients were not randomized and clinical care was not standardized, so that selection bias and the effects of unmeasured confounding variables cannot be excluded. For example, patients in the general anesthesia group had slightly larger tumors, smaller margins, and higher chemotherapy rates than patients in the paravertebral group, factors which could affect mortality, although these differences did not reach statistical significance. Relevant information such as the amount of morphine given and the type of chemotherapy employed in each group was not available in the records. Thus, this study should be viewed as generating a hypothesis and an estimated effect size for future large randomized controlled trials, which are being planned and which will require several years for execution and analysis. However, even a smaller effect size would be clinically important, making this hypothesis an important one to pursue, especially given its biological plausibility according to the arguments presented above.

In summary, we observed a substantial reduction in tumor recurrence and metastases when breast cancer surgery was performed with paravertebral anesthesia and analgesia. Cancer surgery releases tumor cells into surrounding healthy tissue and into the systemic circulation. We speculate that whether these cells become established as recurrent cancer or metastases is likely to depend on immune competence in the immediate perioperative period. Regional anesthesia and analgesia may help preserve immune function by attenuating the surgical stress response and diminishing the need for opioids. Although limited by its retrospective design, our study suggests that prospective trials evaluating the effects of regional analgesia and morphine sparing on cancer recurrence are warranted.

\section{References}

1. Dixon, M. ABC of Breast Diseases. 2nd Edition. 4. BMJ Publishing; London: 2000. p. 75-80. 
2. Buggy DJ, Smith G. Epidural anaesthesia and analgesia: better outcome after major surgery? Growing evidence suggests so. BMJ 1999;319:530-1. [PubMed: 10463878]

3. Ben-Eliyahu S. The price of anticancer intervention. Does surgery promote metastasis? Lancet Oncol 2002;3:578-9. [PubMed: 12233735]

4. Kehlet, H. Modification of responses to surgery by neural blockade: clinical implications In: Neural blockade in clinical anesthesia and pain management. In: Cousins, M.; Bridenbough, M., editors. 5. Lippincott-Raven; Philadelphia: 1998. p. 129-75.

5. Karmakar MK. Thoracic paravertebral block. Anesthesiology 2001;95:771-80. [PubMed: 11575553]

6. O'Riain SC, Buggy DJ, Kerin MJ, Watson RW, Moriarty DC. Inhibition of the stress response to breast cancer surgery by regional anesthesia and analgesia does not affect vascular endothelial growth factor and prostaglandin E2. Anesth Analg 2005;100:244-9. [PubMed: 15616085]

7. Sacerdote P, Bianchi M, Gaspani L, Manfredi B, Maucione A, Terno G, Ammatuna M. Panerai AE: The effects of tramadol and morphine on immune responses and pain after surgery in cancer patients. Anesth Analg 2000;90:1411-4. [PubMed: 10825330]

8. Yeager MP, Colacchio TA, Yu CT, Hildebrandt L, Howell AL, Weiss J, Guyre PM. Morphine inhibits spontaneous and cytokine-enhanced natural killer cell cytotoxicity in volunteers. Anesthesiology 1995;83:500-8. [PubMed: 7661350]

9. Bar-Yosef S, Melamed R, Page GG, Shakhar G, Shakhar K, Ben-Eliyahu S. Attenuation of the tumorpromoting effect of surgery by spinal blockade in rats. Anesthesiology 2001;94:1066-73. [PubMed: 11465599]

10. Ben-Eliyahu S, Shakhar G, Rosenne E, Levinson Y, Beilin B. Hypothermia in barbiturate-anesthetized rats suppresses natural killer cell activity and compromises resistance to tumor metastasis: a role for adrenergic mechanisms. Anesthesiology 1999;91:732-40. [PubMed: 10485785]

11. Page GG, Blakely WP, Ben-Eliyahu S. Evidence that postoperative pain is a mediator of the tumorpromoting effects of surgery in rats. Pain 2001;90:191-9. [PubMed: 11166986]

12. Eden P, Ritz C, Rose C, Ferno M, Peterson C. "Good Old" clinical markers have similar power in breast cancer prognosis as microarray gene expression profilers. Eur J Cancer 2004;40:1837-41. [PubMed: 15288284]

13. Baum M, Demicheli R, Hrushesky W, Retsky M. Does surgery unfavourably perturb the "natural history" of early breast cancer by accelerating the appearance of distant metastases? Eur J Cancer 2005;41:508-15. [PubMed: 15737554]

14. Burke JF. The effective period of preventive antibiotic action in experimental incisions and dermal lesions. Surgery 1961;50:161-168. [PubMed: 16722001]

15. Kurz A, Sessler DI, Lenhardt R. Perioperative normothermia to reduce the incidence of surgicalwound infection and shorten hospitalization. Study of Wound Infection and Temperature Group. N Engl J Med 1996;334:1209-15. [PubMed: 8606715]

16. Wong IH, Lau WY, Leung T, Yeo W, Johnson PJ. Hematogenous dissemination of hepatocytes and tumor cells after surgical resection of hepatocellular carcinoma: a quantitative analysis. Clin Cancer Res 1999;5:4021-7. [PubMed: 10632334]

17. Greif R, Akca O, Horn EP, Kurz A, Sessler DI. Supplemental perioperative oxygen to reduce the incidence of surgical-wound infection. Outcomes Research Group. N Engl J Med 2000;342:161-7. [PubMed: 10639541]

18. Shapiro J, Jersky J, Katzav S, Feldman M, Segal S. Anesthetic drugs accelerate the progression of postoperative metastases of mouse tumors. J Clin Invest 1981;68:678-85. [PubMed: 7276167]

19. van de Vijver MJ, He YD, van't Veer LJ, Dai H, Hart AA, Voskuil DW, Schreiber GJ, Peterse JL, Roberts C, Marton MJ, Parrish M, Atsma D, Witteveen A, Glas A, Delahaye L, van der Velde T, Bartelink H, Rodenhuis S, Rutgers ET, Friend SH, Bernards R. A gene-expression signature as a predictor of survival in breast cancer. N Engl J Med 2002;347:1999-2009. [PubMed: 12490681]

20. Brand JM, Kirchner H, Poppe C, Schmucker P. The effects of general anesthesia on human peripheral immune cell distribution and cytokine production. Clin Immunol Immunopathol 1997;83:190-4. [PubMed: 9143381]

21. Markovic SN, Knight PR, Murasko DM. Inhibition of interferon stimulation of natural killer cell activity in mice anesthetized with halothane or isoflurane. Anesthesiology 1993;78:700-6. [PubMed: 8466070] 
22. Gupta K, Kshirsagar S, Chang L, Schwartz R, Law PY, Yee D, Hebbel RP. Morphine stimulates angiogenesis by activating proangiogenic and survival-promoting signaling and promotes breast tumor growth. Cancer Res 2002;62:4491-8. [PubMed: 12154060] 


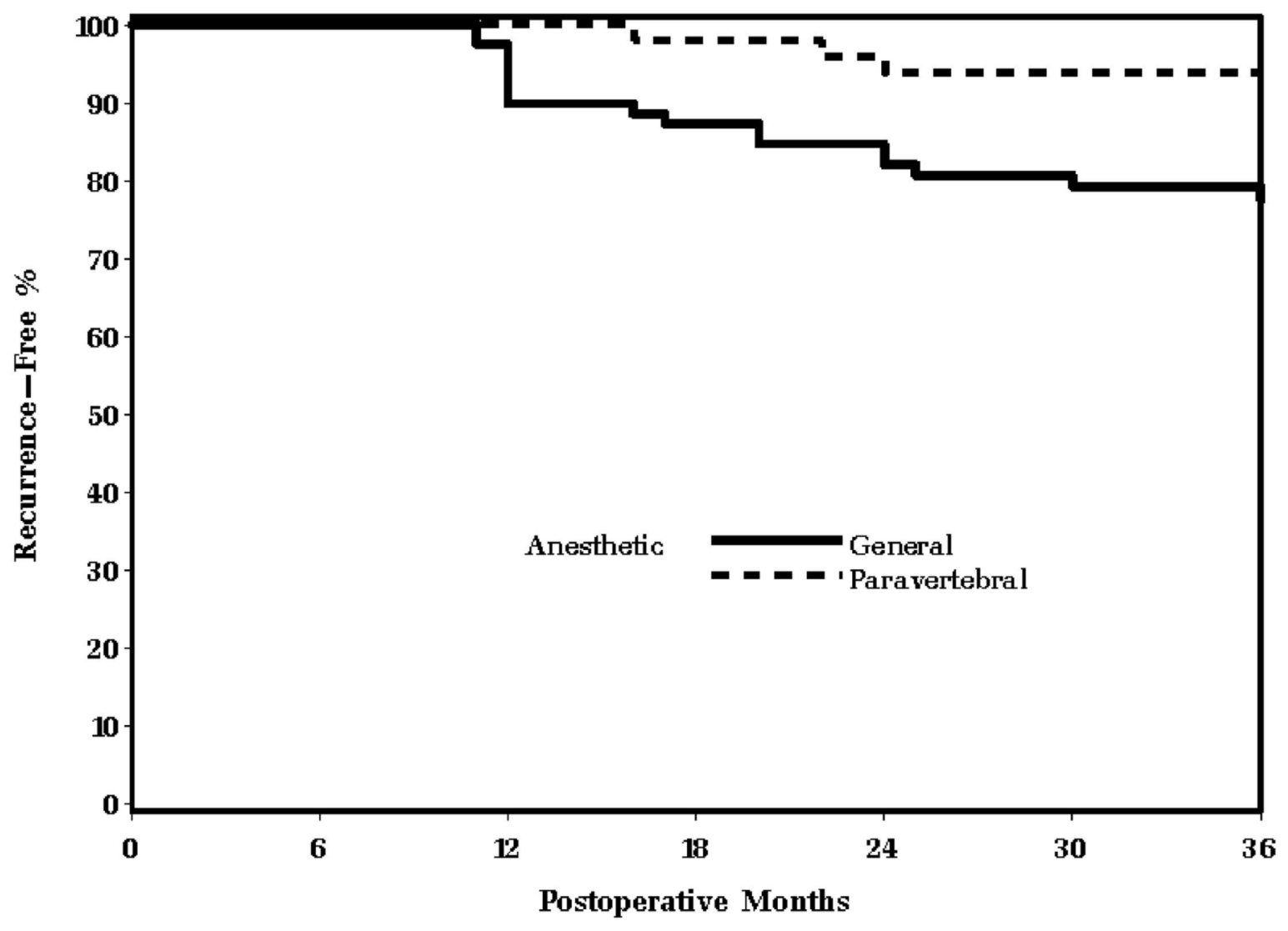

Fig 1.

Univariable association between paravertebral block and cancer recurrence, $P=0.013$ log-rank test. The association remained significant $(P=0.012)$ in a multivariable model adjusting for histological grade and number of axillary nodes. 


\section{Anesthetic and surgical factors}

Table 1

Likert scale $0=$ no pain, $10=$ worse pain imaginable

Data presented as medians [interquartile range] and compared by Mann-Whitney tests.

P-value not meaningful; see survival analysis results 
Table 2

Breast cancer details

\begin{tabular}{|c|c|c|c|}
\hline & Paravertebral $(\mathrm{n}=\mathbf{5 0})$ & General Anesthesia $(n=79)$ & $P$ \\
\hline Tumor size at histology, cm & $4.0 \pm 4.9$ & $4.6 \pm 7.4$ & $0.19^{\S}$ \\
\hline Histological Grade & & & $0.16^{\S}$ \\
\hline Grade I & $8(16 \%)$ & $8(10 \%)$ & \\
\hline Grade II & $21(42 \%)$ & $28(36 \%)$ & \\
\hline Grade III & $21(42 \%)$ & $42(54 \%)$ & \\
\hline Lymph node status & & & $0.94^{\S}$ \\
\hline Stage 1 & $22(58 \%)$ & $35(57 \%)$ & \\
\hline Stage 2 & $4(11 \%)$ & $8(13 \%)$ & \\
\hline Stage 3 & $12(31 \%)$ & $19(30 \%)$ & \\
\hline Nottingham Prognostic Index & $4.5 \pm 1.4$ & $4.7 \pm 1.7$ & $0.38^{\dagger}$ \\
\hline Estrogen receptor positive & $38(76 \%)$ & $46(59 \%)$ & 0.20 \\
\hline Tumor margins after excision, cm & $1.5 \pm 0.8$ & $1.1 \pm 0.7$ & 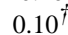 \\
\hline Chemotherapy received & $24(51 \%)$ & $43(58 \%)$ & 0.45 \\
\hline Radiotherapy received & $36(77 \%)$ & $60(81 \%)$ & 0.55 \\
\hline Chemotherapy \& radiotherapy received & $22(47 \%)$ & $39(52 \%)$ & 0.48 \\
\hline Endocrine therapy received & $27(54 \%)$ & $44(56 \%)$ & 0.85 \\
\hline
\end{tabular}

All data are means \pm SDs or number of patients (\%).

*

* Chi-Square test unless noted;

$\dagger_{\text {t-test }}$

$\S_{\text {Mann-Whitney test }}$ 


\section{Table 3}

Cancer recurrence

\begin{tabular}{|c|c|c|}
\hline & Paravertebral $(\mathbf{n}=50)$ & General Anesthesia $(n=79)$ \\
\hline Crude Recurrence & $3(6 \%)$ & $19(24 \%)$ \\
\hline $\begin{array}{l}\text { Percent of patients recurrence-free at } 24 \text { months [95\% } \\
\text { CI] }\end{array}$ & $94[87,100]$ & $82[74,91]^{*}$ \\
\hline $\begin{array}{l}\text { Percent of patients recurrence-free at } 36 \text { months [ } 95 \% \\
\text { CI] }\end{array}$ & $94[87,100]$ & $77[68,87]^{\dagger}$ \\
\hline \multicolumn{3}{|l|}{$\begin{array}{l}\text { C1] } \\
\text { Recurrence location }\end{array}$} \\
\hline Local or axillary nodes & 1 & 11 \\
\hline Liver metastasis & 1 & 3 \\
\hline Bone metastasis & 1 & 3 \\
\hline Lung metastasis & 0 & 2 \\
\hline
\end{tabular}

Data are number (percent) or Kaplan Meier survival estimate [95\% CI]

P $=0.038$ comparing groups on Kaplan-Meier estimates at 24 months (z-test)

${ }^{\dagger} \mathrm{P}=0.007$ comparing groups on Kaplan-Meier estimates at 36 months (z-test) 